\title{
Rare earth fluorescence in NASICON type phosphate glass, $\mathrm{Na}_{3} \mathrm{TiZnP}_{\mathbf{3}} \mathrm{O}_{12}$
}

\author{
G VIJAYA PRAKASH, P NACHIMUTHU, $M$ VITHAL $^{\dagger}$ and R JAGANNATHAN* \\ School of Chemistry, University of Hyderabad, Hyderabad 500046 , India \\ 'Department of Chemistry, Osmania University, Hyderabad 500007 , India
}

MS received 15 October 1998; revised 22 January 1999

\begin{abstract}
The emission spectra of $\mathrm{Pr}^{3+}, \mathrm{Eu}^{3+}$ and $\mathrm{Dy}^{3+}$ doped in NASICON type phosphate glass, $\mathrm{Na}_{3} \mathrm{TiZnP}_{3} \mathrm{O}_{12}$ (NTZP) are studied. The dopant rare earth ions occupy sites with 8-9 coordination in a highly covalent environment. For $\mathrm{Pr}^{3+}$ ion, calculated and observed branching ratios for lasing transitions ${ }^{3} \boldsymbol{P}_{0} \rightarrow{ }^{3} H_{4,6}$ agree well and found to be 0.64 and 0.24 , respectively. The emissions of $\mathrm{Pr}^{3+}$ show strong temperature dependence on account of Boltzmann population of the higher excited states at room temperature. The excitation spectrum of $\mathrm{Eu}^{3+}$ gives rise to phonon assisted side band for ${ }^{5} D_{2} \leftarrow{ }^{7} F_{0}$ transition at higher energy side with a phonon energy maximum of $1022 \mathrm{~cm}^{-1}$ and an electron phonon coupling strength $(\mathrm{g})$ of 0.018 . The value of phonon energy maximum agrees with infrared spectral data. The results show that observation of high energy emissions in phosphate glasses require much higher $g$ values. The red/orange and yellow/blue transitions of $\mathrm{Eu}^{3+}$ and $\mathrm{Dy}^{3+}$, respectively show that the $\mathrm{Eu}^{3+}$ occupy more distorted site than $\mathrm{Dy}^{3+}$.
\end{abstract}

Keywords. Rare earths; emission spectra; excitation spectra; phonon side band; electron phonon coupling strength; branching ratio.

\section{Introduction}

Optical studies of rare earth ions in glass systems are of considerable interest due to their potential applications in lasing materials, holographic gratings and hole burning devices (Durville et al 1987; Caird et al 1991; Yzexing 1995; Cho et al 1996; Menezes et al 1997). Phosphate glasses belonging to the family of NASICON (acronym for the crystalline sodium superionic conductor based on $\left.\mathrm{Na}_{1+x} \mathrm{Zr}_{2} \mathrm{P}_{3-x} \mathrm{Si}_{x} \mathrm{O}_{12}\right)$ attract special attention as they exhibit several features such as preparation of glasses with systematic variation in composition, low value for maximum phonon energy leading to low nonradiative loss during emission and scope for preparing neat glasses by sol-gel route (Hagman and Kierkegaard 1968; Sobha and Rao 1995a, 1996a, b). They have the general formula $\mathrm{A}_{m} \mathrm{~B}_{n} \mathrm{P}_{3} \mathrm{O}_{12}$, where $\mathrm{A}$ is an alkali or alkaline earth metal ion and $B$ is a tri; tetra or pentavalent metal ion (Sobha and Rao 1995b). It would therefore be of interest to investigate site preferences for the dopant rare earth ions in these glass matrices and study their emission properties as influenced by hypersensitivity, covalency, site asymmetry and the local structure. The present paper deals with the fluorescence properties of $\mathrm{Pr}^{3+}, \mathrm{Eu}^{3+}$ and $\mathrm{Dy}^{3+}$ ions doped in $\mathrm{Na}_{3} \mathrm{TiZnP}_{3} \mathrm{O}_{12}$ (hereafter NTZP) glass.

\footnotetext{
*Author for correspondence
}

\section{Experimental}

The glass compositions, $\mathrm{Na}_{3} \mathrm{TiZnP}_{3} \mathrm{O}_{\mathrm{i} 2}$ (NTZP) and 2 $w t \% \mathrm{Ln}^{3+}\left(\mathrm{Pr}^{3+}, \mathrm{Eu}^{3+}\right.$ and $\left.\mathrm{Dy}^{3+}\right)$, were prepared using stoichiometric amounts of $\mathrm{NH}_{4} \mathrm{H}_{2} \mathrm{PO}_{4}, \mathrm{TiO}_{2}, \mathrm{ZnO}, \mathrm{NaNO}_{3}$ (Analytical grade) and $2 \mathrm{wt} \% \mathrm{Ln}_{2} \mathrm{O}_{3}$ (Indian Rare Earths Limited or Aldrich Chemical Co., USA) as starting materials by standard melt quenching procedure. The absorption spectra were recorded on a JASCO 7800 UV-VIS spectrophotometer and Hitachi 3310 spectrophotometer using undoped glass as reference. The excitation and emission spectra were recorded on a Hitachi 3010 spectrofluorimeter using appropriate filters. Densities were measured by Archemedes principle using toluene as immersion liquid. Refractive index measurements were carried out by Brewster angle setup consisting of $\mathrm{He}-\mathrm{Ne}$ laser and a diode detector. Density (d) and refractive index $(\eta)$ of the present glass were found to be $3.18 \pm 0.04 \mathrm{~g} \cdot \mathrm{cm}^{-3}$ and $1.660 \pm 0.002$, respectively.

\section{Results}

From the absorption spectra of $2 \mathrm{wt} \% \mathrm{Pr}^{3+}$ and $\mathrm{Dy}^{3+}$ doped in NTZP glass samples, oscillator strengths of the observed transitions were obtained by the area method (table 1) (Carnall et al 1978). The Judd-Ofelt parameters $\left(\Omega_{\lambda=2,4,0}\right)$ are evaluated from the oscillator strengths of the observed transitions using the procedure described 
Table 1. Observed and calculated oscillator strengths for $\mathrm{Pr}^{3+}$ and $\mathrm{Dy}^{3+}$ ions in NTZP glass.

\begin{tabular}{|c|c|c|c|c|c|c|c|}
\hline \multirow{2}{*}{$\begin{array}{l}\mathrm{Pr}^{3+} \\
\text { transition } \\
\text { from }{ }^{3} H_{4}\end{array}$} & \multirow{2}{*}{$\begin{array}{l}\text { Energy } \\
\left(\mathrm{cm}^{-1}\right)\end{array}$} & \multicolumn{2}{|c|}{$P\left(10^{-6}\right)$} & \multirow{2}{*}{$\begin{array}{l}\text { Dy }{ }^{3+} \\
\text { transition from } \\
{ }^{6} H_{15 / 2}\end{array}$} & \multirow{2}{*}{$\begin{array}{l}\text { Energy } \\
\left(\mathrm{cm}^{-1}\right)\end{array}$} & \multicolumn{2}{|c|}{$P\left(10^{-6}\right)$} \\
\hline & & obs & $\mathrm{cal}$ & & & obs & cal \\
\hline${ }^{3} F_{2}$ & 5197 & $2 \cdot 87$ & $2 \cdot 56$ & ${ }^{4} G_{11 / 2}{ }^{*}$ & 23596 & $0 \cdot 31$ & 0.34 \\
\hline${ }^{3} F_{3}$ & 6554 & 5.05 & $6 \cdot 17$ & ${ }^{4} I_{15 / 2} *$ & 22109 & 0.70 & 0.43 \\
\hline${ }^{3} F_{4}$ & 7092 & 1.56 & 3.66 & ${ }^{4} F_{9 / 2}$ & 21168 & 0.44 & 0.23 \\
\hline${ }^{1} G_{4}$ & 9038 & 0.50 & 0.28 & ${ }^{6} F_{3 / 2}$ & 13324 & 0.20 & 0.17 \\
\hline${ }^{\prime} D_{2}$ & 16926 & 1.41 & 1.08 & ${ }^{6} F_{5 / 2}$ & 12500 & $1 \cdot 16$ & 0.92 \\
\hline${ }^{3} P_{6}$ & 20734 & 2.61 & 2.48 & ${ }^{6} F_{7 / 2},{ }^{6} H_{7 / 2}$ & 11164 & 1.99 & $3 \cdot 18$ \\
\hline${ }^{3} P_{1}$ & 21267 & 3.58 & 3.81 & ${ }^{6} F_{9 / 2},{ }^{6} H_{11 / 2}$ & 9259 & $7 \cdot 13$ & $6 \cdot 71$ \\
\hline \multirow[t]{3}{*}{${ }^{3} P_{2}^{*}$} & 22512 & 7.47 & 3.57 & ${ }^{6} H_{9 / 2},{ }^{6} F_{11 / 2}{ }^{*}$ & 8032 & 12.71 & $12 \cdot 81$ \\
\hline & & & & ${ }^{6} H_{11 / 2}$ & 6035 & 2.83 & 1.44 \\
\hline & \multicolumn{3}{|c|}{$\mathrm{rms}=1.62$} & & \multicolumn{3}{|c|}{$\mathrm{rms}=0.64$} \\
\hline
\end{tabular}

*Hypersensitive.

Table 2. Judd-Ofelt parameters for $\mathrm{Pr}^{3+}$ and $\mathrm{Dy}^{3+}$ ions in NTZP glass.

\begin{tabular}{lccc}
\hline Lanthanide ion & $\Omega_{2}\left(10^{-20} \mathrm{~cm}^{2}\right)$ & $\Omega_{4}\left(10^{-20} \mathrm{~cm}^{2}\right)$ & $\Omega_{6}\left(10^{-20} \mathrm{~cm}^{2}\right)$ \\
\hline $\mathrm{Pr}^{3+}$ & $2.09 \pm 0.39$ & $3.95 \pm 0.53$ & $3.29 \pm 0.47$ \\
& $(1.05 \pm 1.29)^{*}$ & $(3.79 \pm 1.83)$ & $(5.40 \pm 1.83)$ \\
$\mathrm{Dy}^{3+}$ & $7.04 \pm 0.85$ & $9.55 \pm 0.60$ & $2.06 \pm 1.27$ \\
\hline
\end{tabular}

*The values in parenthesis are deduced by including hypersensitive transition ${ }^{3} \mathrm{P}_{2} \rightarrow{ }^{3} \mathrm{H}_{4}$.

earlier (Reisfeld 1975; Srikanth Sarma et al 1992; Nachimuthu and Jagannathan 1995a; Vijaya Prakash et al 1998). The values thus obtained are shown in table 2 for $\mathrm{Pr}^{3+}$ and $\mathrm{Dy}^{3+}$ ions. No attempt was made to evaluate the Judd-Ofelt parameters for $\mathrm{Eu}^{3+}$ as the number of transitions observed in the absorption spectrum are limited and masked by the absorption edge characteristic of these glasses. The Judd-Ofelt parameters $\left(\Omega_{\lambda}\right)$ are used to calculate radiative parameters, viz. transition probabilities (A), radiative life times $\left(\tau_{R}\right)$, branching ratios $\left(\beta_{\text {cal }}\right)$ and stimulated emission cross sections $\left(\sigma_{\mathrm{p}}\right)$ using the relevant relationships given in our earlier works and are shown in table 3 (Nachimuthu and Jagannathan 1995a).

The emission spectra of $\mathrm{Pr}^{3+}, \mathrm{Eu}^{3+}$ and $\mathrm{Dy}^{3+}$ are recorded choosing the appropriate excitation wavelengths. Figure 1 shows the emission spectra of $\mathrm{Pr}^{3+}$ doped in NTZP at room temperature and $77 \mathrm{~K}$. The emission spectrum of $\mathrm{Eu}^{3+}$ doped in NTZP is shown in figure 2 along with the phonon assisted side band observed in the excitation spectrum monitored at ${ }^{5} D_{0} \rightarrow{ }^{7} F_{2}$. Figure 3 shows the room temperature emission spectrum of $\mathrm{Dy}^{3+}$ doped in NTZP. The observed branching ratios are obtained from the relative intensities of the emission transitions. The emission transitions in the near infrared region are assumed to be comparatively negligible. The values of radiative parameters thus obtained are also given in table 3 .

\section{Discussion}

$\mathrm{Pr}^{3+}$ : The ground state of $\mathrm{Pr}^{3+}\left(4 f^{2}\right)$ is ${ }^{3} H_{4}$. The $\Omega_{\lambda}$ values obtained including the oscillator strengths of the hypersensitive transitions lead to large errors. $\mathrm{Pr}^{3+}$ ion is known to show exceptional values for the Judd-Ofelt parameters due to hypersensitive transition ${ }^{3} \mathrm{P}_{2} \rightarrow{ }^{3} \mathrm{H}_{4}$ (Bunvel et al 1992). Hence the values, excluding this transition, which are more reliable for comparison and discussion, are also obtained (table 2). Among the JuddOfelt parameters, $\boldsymbol{\Omega}_{\mathbf{2}}$ is the most sensitive to the nature of the matrix. Fluoride glasses which give rise to most ionic matrix have the least $\Omega_{2}$ values ranging from 0.06 
to 0.84 (in units of $10^{-20} \mathrm{~cm}^{2}$ ), (Bunvel et al 1992; Binnemans and Gorller-Walrand 1998). The much higher $\Omega_{2}$ values obtained for the present glass shows that the phosphate ligands provide highly covalent environment. An alternate procedure of obtaining Judd-Ofelt parameters, $\boldsymbol{\Omega}_{\lambda}$ using the experimental branching ratios of ${ }^{3} P_{0}$ level has been recommended by Bunvel et al (1992). This procedure may however introduce errors due to the uncertainties in branching ratios obtained. The $\Omega_{\text {, values }}$ obtained by this method along with the experimental and calculated oscillator strengths are reproduced in appendix. This procedure led to $\Omega_{2}$ value significantly lower and more characteristic of ionic systems such as fluoride glasses. The values obtained by the earlier procedure which are characteristic of covalent systems such as phosphate glasses may therefore be considered as more reliable. It is seen from table 3 that the calculated and observed branching ratios for the lasing transitions ${ }^{3} P_{0} \rightarrow{ }^{3} H_{4.6}$ agree quite well showing that the mechanism leading to non-radiative decays are not very much operative unlike in the case of the weak ${ }^{3} P_{0} \rightarrow{ }^{3} F_{2}$ laser transition. The calculated $\tau_{\mathrm{R}}$ values give the upper limits in all the cases as the actual values are influenced by multiphonon relaxation and cross relaxations between the adjacent ions (Bunvel et al 1992). The large radiative decay probabilities observed for the ${ }^{3} P_{0} \rightarrow{ }^{3} H_{4,6}$ and ${ }^{3} P_{0} \rightarrow{ }^{3} F_{2}$ laser transitions for $\mathrm{Pr}^{3+}$ are also in order with the strong interaction of rare earth ions with local sites as suggested by the large electron phonon coupling strength from the phonon side band for $\mathrm{Eu}^{3+}$ ions in this glass matrix as discussed later.

The asymmetric emission spectrum of $\mathrm{Pr}^{3+}$ in the region

Table 3. Calculated and observed radiative parameters for $\mathrm{Pr}^{3+}, \mathrm{Eu}^{3+}$ and $\mathrm{Dy}^{3+}$ ions in NTZP glass.

\begin{tabular}{|c|c|c|c|c|c|c|c|}
\hline Transition & $\begin{array}{c}v \\
\left(\mathrm{~cm}^{-1}\right)\end{array}$ & $\underset{\left(s^{-1}\right)}{A}$ & $\begin{array}{l}\beta_{\mathrm{R}} \\
\text { (cal) }\end{array}$ & $\begin{array}{c}\beta_{\mathrm{R}} \\
\text { (obs) }\end{array}$ & $\begin{array}{c}\boldsymbol{\tau}_{\mathrm{R}} \\
(\mu \mathrm{s})\end{array}$ & $\begin{array}{c}\sigma \mathrm{p} \times 10^{-22} \\
\left(\mathrm{~cm}^{2}\right)\end{array}$ & $\begin{array}{c}\text { Ratio of } \\
\text { areas }\end{array}$ \\
\hline \multicolumn{8}{|l|}{ Praseodymium (III)* } \\
\hline${ }^{3} P_{0} \rightarrow{ }^{3} H_{4}$ & 20534 & 16885 & 0.66 & $\begin{array}{c}0.64 \\
(0.83)\end{array}$ & 39.4 & 257 & \\
\hline${ }^{3} P_{0} \rightarrow{ }^{3} H_{5}$ & 18182 & - & - & $\begin{array}{c}0.08 \\
(0.08)\end{array}$ & & - & \\
\hline${ }^{3} P_{0} \rightarrow{ }^{3} H_{6}$ & 16239 & 4996 & 0.20 & $\begin{array}{c}0.24 \\
(0.07)\end{array}$ & & 156 & \\
\hline${ }^{3} P_{0} \rightarrow{ }^{3} F_{2}$ & 15537 & 3466 & 0.14 & $\begin{array}{c}0.03 \\
(0.01)\end{array}$ & & 281 & \\
\hline${ }^{3} P_{1} \rightarrow{ }^{3} H_{5}$ & 19387 & 11069 & 0.34 & - & 30.7 & 291 & \\
\hline${ }^{1} D_{2} \rightarrow{ }^{3} H_{4}$ & 16726 & 961 & 0.41 & - & 415 & - & \\
\hline \multicolumn{8}{|l|}{ Europium (III) } \\
\hline${ }^{5} D_{0} \rightarrow{ }^{7} F_{0}$ & 17271 & - & - & 0.03 & - & & \\
\hline${ }^{5} D_{0} \rightarrow{ }^{7} F_{1}$ & 16915 & - & - & 0.28 & - & - & \\
\hline${ }^{5} D_{0} \rightarrow{ }^{7} F_{2}$ & 16329 & - & - & 0.67 & - & - & \\
\hline${ }^{5} D_{0} \rightarrow{ }^{7} F_{3}$ & 15356 & - & - & 0.009 & - & - & \\
\hline${ }^{5} D_{0} \rightarrow{ }^{7} F_{4}$ & 14310 & - & - & 0.008 & - & - & \\
\hline $\mathrm{R} / \mathrm{O}=\left({ }^{5} D_{0} \rightarrow{ }^{7} F_{2}\right) /\left({ }^{5} D_{0} \rightarrow{ }^{7} F_{1}\right)$ & & & & & & & $2 \cdot 38$ \\
\hline \multicolumn{8}{|l|}{ Dysprosium (III) } \\
\hline${ }^{4} F_{9 / 2} \rightarrow{ }^{6} H_{15 / 2}$ & 21151 & 294 & 0.19 & 0.58 & 680 & $4 \cdot 3$ & \\
\hline${ }^{4} F_{9 / 2} \rightarrow{ }^{6} H_{13 / 2}$ & 17666 & 1008 & 0.68 & 0.42 & & $37 \cdot 8$ & \\
\hline${ }^{4} F_{9 / 2} \rightarrow{ }^{6} H_{11 / 2}$ & 15133 & 92 & 0.06 & 0.02 & & - & \\
\hline${ }^{4} F_{9 / 2} \rightarrow{ }^{6} H_{9 / 2},{ }^{6} F_{11 / 2}$ & 13136 & 30 & 0.02 & - & & - & \\
\hline${ }^{4} F_{9 / 2} \rightarrow{ }^{6} H_{7 / 2},{ }^{6} F_{9 / 2}$ & 11909 & 25 & 0.02 & - & & - & \\
\hline${ }^{4} F_{9 / 2} \rightarrow{ }^{6} H_{5 / 2},{ }^{6} F_{7 / 2}$ & 10004 & $10 \cdot 6$ & 0.007 & - & & - & \\
\hline${ }^{4} F_{9 / 2} \rightarrow{ }^{6} F_{9 / 2}$ & 8668 & $10 \cdot 1$ & 0.007 & - & & - & \\
\hline$Y / B=\left({ }^{4} F_{9 / 2} \rightarrow{ }^{6} H_{15 / 2}\right) /\left({ }^{4} F_{9 / 2} \rightarrow{ }^{6} H_{13 / 2}\right)$ & & & & & & & 0.71 \\
\hline
\end{tabular}

*Parenthesis values indicate the branching ratios of $\mathrm{Pr}^{3+}$ at $77 \mathrm{~K}$. 
$517-570 \mathrm{~nm}$ is composed of a major peak due to ${ }^{3} P_{1} \rightarrow{ }^{3} H_{5}$ and a minor peak due to ${ }^{3} P_{0} \rightarrow{ }^{3} H_{5}$ (figure 1 ). The latter is a strong dipole forbidden transition and its presence indicates significant $J-J$ mixing (Strek et al 1985). The relative emission intensities of $\mathrm{Pr}^{3+}$ originating from ${ }^{3} P_{1}$ level significantly decreases on cooling the sample to $77 \mathrm{~K}$ (figure 1). The appearance of ${ }^{3} P_{1}$ emission at higher temperature is due to the enhanced Boltzmann population of the higher Stark components of the ground state $\left({ }^{3} H_{4}\right)$ facilitating direct pumping to ${ }^{3} P_{1}$ and/or by the thermal excitation from ${ }^{3} P_{0}$ to ${ }^{3} P_{1}$ (Strek et al 1985). The possible reasons for the disagreement in the observed and calculated branching ratios for ${ }^{3} P_{0} \rightarrow{ }^{3} H_{6}$ and ${ }^{3} P_{0} \rightarrow{ }^{3} F_{2}$ may be due to interference of other emission transitions from ${ }^{3} P_{1}$ level occurring in the same regions (Nachimuthu and Jagannathan 1995a; Zhao and Flemming 1997).

$E u^{3+}:$ The ground state of $\mathrm{Eu}^{3+}\left(4 f^{6}\right)$ is ${ }^{7} F_{0}$. The excitation spectrum of $\mathrm{Eu}^{3+}$ monitored at ${ }^{5} D_{0} \rightarrow{ }^{7} F_{2}$ shows the phonon assisted side band for ${ }^{5} D_{2} \leftarrow{ }^{7} F_{0}$ transition at higher energy side (figure 2). The energy between the pure electronic state and the phonon side band corresponds to the phonon energy, $\hbar \omega$ (Toratoni et al 1982). The $\hbar \omega$ value thus obtained $\left(1022 \mathrm{~cm}^{-1}\right)$ is in good agreement with the infrared absorption maximum, $h v\left(1024 \mathrm{~cm}^{-1}\right)$, which arises due to the $\mathrm{P}-\mathrm{O}$ vibrational modes (Videau et al 1982; Sobha and Rao 1995b). The value of electron phonon coupling strength $(g=0.018)$, estimated from the ratio of the area under phonon side band and corresponding pure electronic transition is much higher than $g$-values for fluoride and oxyfluoride glasses. This is due to the strong covalent bonding of rare earth ion with the local site and also higher non-radiative decay could be expected in this glass system (Tanabe et al 1992).

The position of ${ }^{5} D_{0} \rightarrow{ }^{7} F_{0}$ of $\mathrm{Eu}^{3+}$ emission is characteristic of the coordination of the $\mathrm{Eu}^{3+}$ ion (Boulon et al 1985). This emission transition is found to be in the range $17,271-17,574 \mathrm{~cm}^{-1}$ suggesting the $8-9$

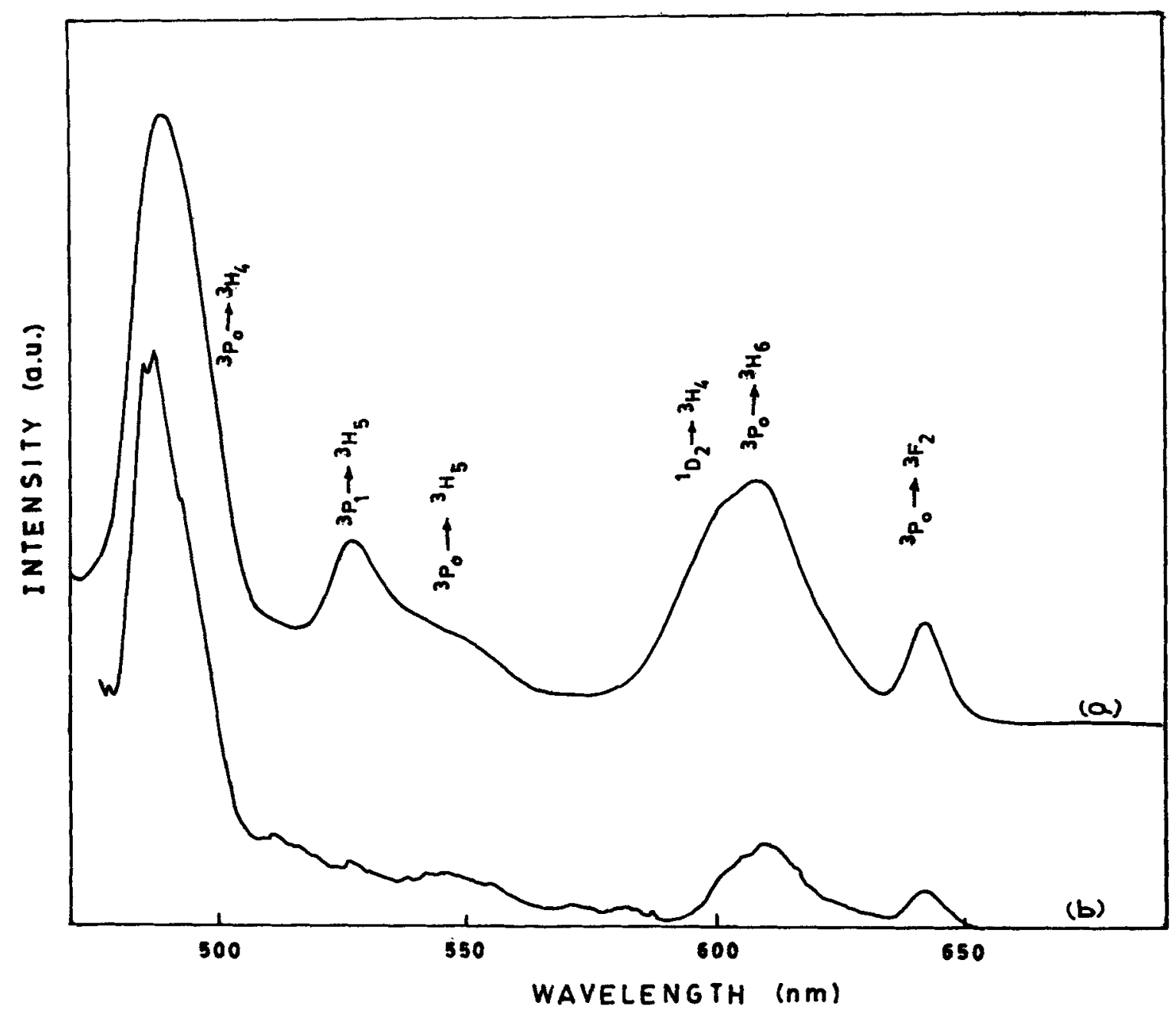

Figure 1. Emission spectra of $2 \mathrm{wt} \% \mathrm{Pr}^{3+}$ doped NTZP glass excited at $446 \mathrm{~nm}$ (a) at room temperature and (b) at $77 \mathrm{~K}$. 
coordination for $\mathrm{Eu}^{3+}$. The room temperature emission spectrum of $\mathrm{Eu}^{3+}$ doped in NTZP glass did not show transitions in the high energy region $(400-520 \mathrm{~nm})$ unlike fluoride, oxyfluoride, germanate and tellurite glasses (Reisfeld 1975; Nachimuthu and Jagannathan 1995b). The high energy transitions are observed if (i) phonon energy maxima for the parent matrices are low and (ii) electron phonon coupling strengths $(g)$ are high. In the case of germanates, tellurites and oxyfluorides, the phonon energy maximum is quite low and even when the coupling strengths are not high, the high energy transitions are observed. In the case of borates for which the phonon energy maximum is high, irrespective of the coupling strengths the high energy transitions are not observed. In the case of lead phosphate glasses, the phonon energy maxima have intermediate values, but the electron-phonon coupling strengths $(g)$ are quite high and therefore the high energy transitions although weak are observed. In the case of NTZP glass, the phonon energy maximum is no doubt intermediate $\left(1024 \mathrm{~cm}^{-1}\right)$ as in the lead phosphate glass but the electron phonon coupling strength also is much less and hence these transitions are not observed.

The emission spectrum shows two prominent transitions viz. ${ }^{5} D_{0} \rightarrow{ }^{7} F_{1}$ (orange) and ${ }^{5} D_{0} \rightarrow{ }^{7} F_{2}$ (red) which appeared around $612 \mathrm{~nm}$ and $591 \mathrm{~nm}$, respectively. The transition ${ }^{5} D_{0} \rightarrow{ }^{7} F_{1}$ is purely magnetic dipole and its intensity is not influenced by metal ligand bonding. On the other hand, ${ }^{5} D_{0} \rightarrow{ }^{3} F_{2}$ which is electric dipole in nature and hypersensitive, is very much influenced by local factors such as Eu-O covalency and symmetry around $\mathrm{Eu}^{3+}$ ion. Their relative intensities $(R / O)$, thus show a systematic change with the nature of the parent glass matrices (Nachimuthu et al 1997). The $R / O$ ratio in the present NTZP glass is found to be 2.38 which is considerably higher than the values observed for the fluoride and oxyfluoride glasses but lower than silicate and borate glasses. It is notable that this ratio is approximately equal to the $R / O$ ratio found in calcium phosphate and calcium alumino phosphate glasses (Nachimuthu 1996). Thus the $R / O$ ratio follows the order

silicate $>$ borate $>\mathrm{NTZP} \cong$ phosphate $>$ oxyfluoride.

$D y^{3+}$ : The ground state of $\mathrm{Dy}^{3+}\left(4 f^{9}\right)$ is ${ }^{6} F_{15 / 2}$. The hypersensitive transition ${ }^{6} F_{11 / 2} \rightarrow{ }^{6} H_{15 / 2}$ of $D^{3+}$ at $1.34 \mu \mathrm{m}$ has potential for fibre amplifiers (Wei et al 1994). The values of Judd-Ofelt parameters are significantly higher than the values reported for fluoride and oxyfluoride glasses (Nachimuthu et al 1997). Unlike $\mathrm{Eu}^{3+}$, the two dominant emission transitions ${ }^{4} F_{9 / 2} \rightarrow{ }^{6} H_{15 / 2}$ and ${ }^{4} F_{9 / 2} \rightarrow{ }^{6} H_{13 / 2}$ of Dy ${ }^{3+}$ are electric dipole one of which, ${ }^{4} F_{9 / 2} \rightarrow{ }^{6} H_{15 / 2}$ (yellow), is hypersensitive (figure 3) (Nachimuthu et al 1997). Su et al (1993) have shown that the ratio of hypersensitive to non-hypersensitive emission transitions of $\mathrm{Eu}^{3+}$, $\left({ }^{5} D_{01} \rightarrow{ }^{7} F_{2}\right) /\left({ }^{5} D_{0} \rightarrow{ }^{7} F_{1}\right)($ i.e. $R / O)$ and $\left({ }^{4} F_{9 / 2} \rightarrow{ }^{6} H_{15 / 2}\right) /$

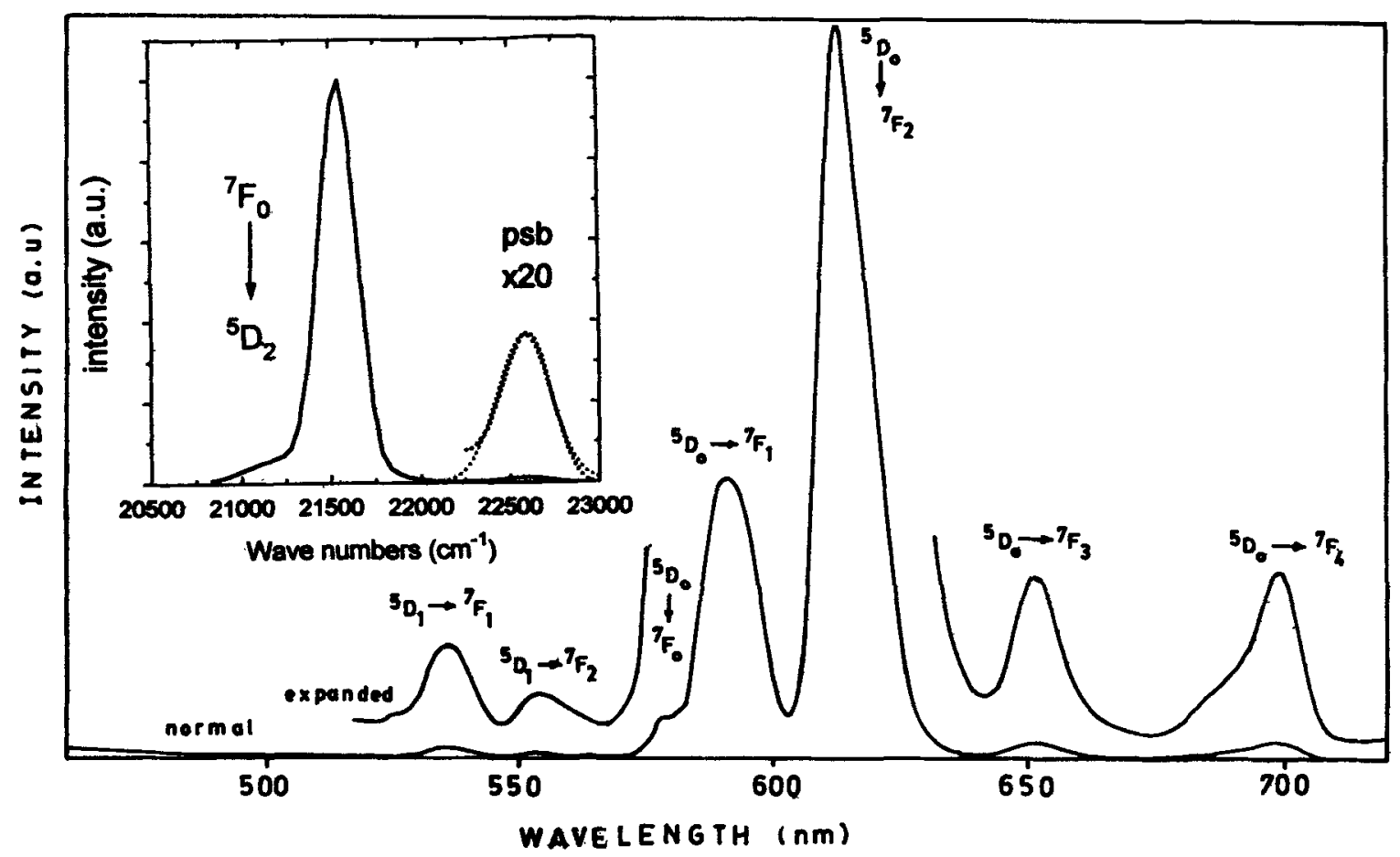

Figure 2. Emission spectrum of $2 \mathrm{wt} \% \mathrm{Eu}^{3+}$ doped NTZP glass excited at $394 \mathrm{~nm}$. Inset shows the phonon side band (PSB) of $\mathrm{Eu}^{3+}$ excitation spectrum monitored at ${ }^{5} D_{0} \rightarrow{ }^{7} F_{2}$. 
$\left({ }^{4} F_{9 / 2} \rightarrow{ }^{6} H_{13 / 2}\right)$ (i.e. $\left.Y / B\right)$ of $\mathrm{Dy}^{3+}$ follow a parallel trend as these ratios are sensitive to the covalency and site asymmetry. The emission transitions of $\mathrm{Eu}^{3+} \mathrm{viz}$. ${ }^{5} D_{0} \rightarrow{ }^{7} F_{1}$ and ${ }^{5} D_{2} \rightarrow{ }^{7} F_{2}$ exhibit strikingly unequal intensity $(R / O=2 \cdot 38)$, indicates that the $\mathrm{Eu}^{3+}$ ion is predominantly situated in highly distorted asymmetric crystal field. On the other hand, the emission spectrum of $\mathrm{Dy}^{3+}$ doped glass shows almost equal intensity $(Y / B=0.71)$ for two dominant emission transitions ${ }^{4} F_{9 / 2} \rightarrow{ }^{6} H_{15 / 2}$ and ${ }^{4} F_{9 / 2} \rightarrow{ }^{6} H_{13 / 2}$ (figure 3). Su et al (1993) have shown that the $Y / B$ ratio for crystalline systems fall in the range 3.0-1.19. Our value lies below this range. This indicates that unlike $\mathrm{Eu}^{3+}$ ions, $\mathrm{Dy}^{3+}$ ions occupy sites which are less distorted. Such a difference may arise due to significant difference in the radii of $\left(\mathrm{Eu}^{3+} 95.0 \mathrm{pm}\right.$ and $\left.\mathrm{Dy}^{3+} 90.8 \mathrm{pm}\right)$ the rare earth ions as we go along the lanthanide series.

The $Y / B$ ratio observed for NTZP glass is higher than borate but lower than oxyfluoride, fluoride and silicate glasses. Thus the order of $Y / B$ is

oxyfluoride $>$ silicate $\cong$ fluoride $>N T Z P \cong$ phosphate $>$ borate.

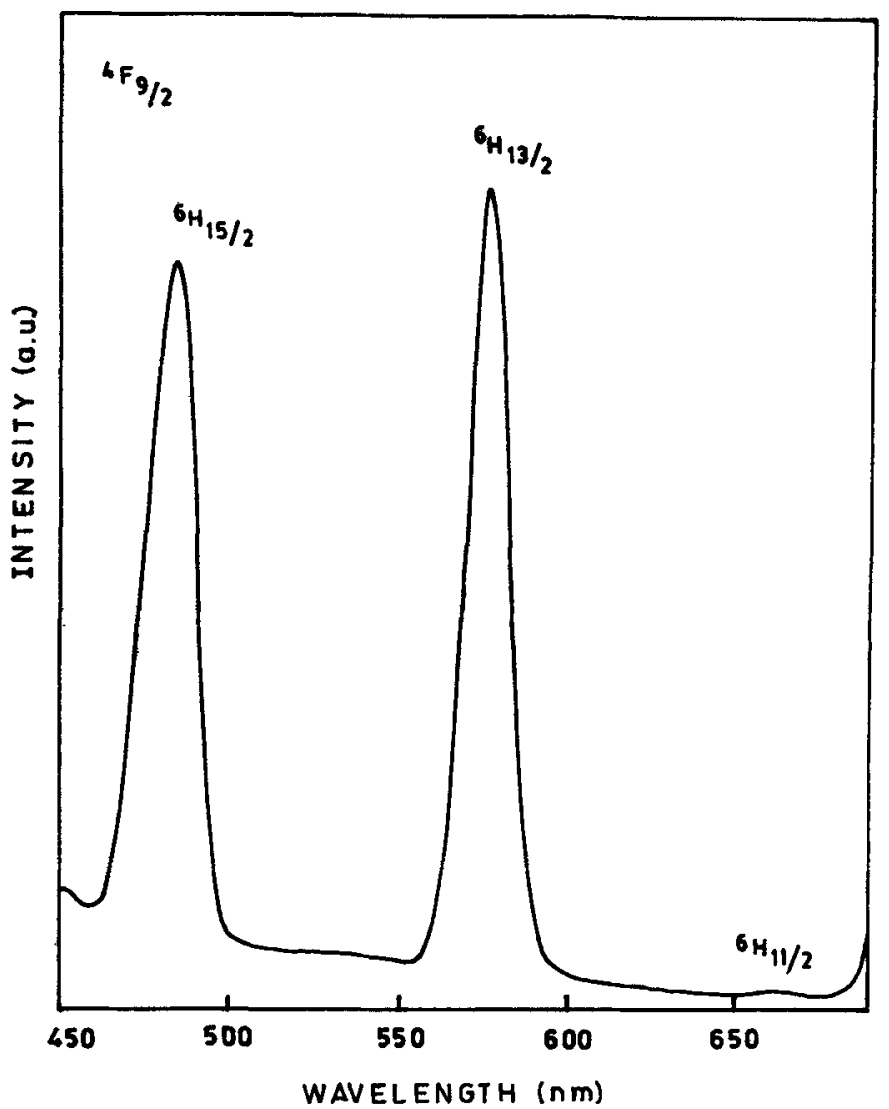

Figure 3. Emission spectrum of $2 \mathrm{wt} \% \mathrm{Dy}^{3+}$ doped NTZP glass excited at $350 \mathrm{~nm}$.
It is observed that $Y / B$ ratio of phosphate and NTZP glasses are approximately same as the network in both the glasses is closely similar.

The differences in the relative trends of the $R / O$ ratio of $\mathrm{Eu}^{3+}$ and $Y / B$ ratio of $\mathrm{Dy}^{3+}$ arise due to the differences in the nature of the transition involved. In $\mathrm{Dy}^{3+}$ both the transitions are electric dipole, one of which $\left({ }^{4} F_{9 / 2} \rightarrow{ }^{6} H_{13 / 2}\right)$ is hypersensitive. Richardson has shown that the intensity calculations of $f-f$ transitions in such cases is affected by the differences in charges and polarizabilities of the ligand atoms in the axial and equatorial positions even when the coordination geometries are similar (Richardson et al 1981).

The $\Omega_{2}$ values for $\mathrm{Dy}^{3+}$ in NTZP glasses are markedly higher than reported for oxyfluoride and fluoride glasses (Nachimuthu et al 1997). This shows that $\mathrm{Dy}^{3+}$ is highly covalently bound in these phosphate glass matrices. The radiative parameters for different transitions for $\mathrm{Dy}^{3+}$ deduced from Judd-Ofelt parameters are given in table 3. The differences between the calculated and observed branching ratio may arise due to errors involved in determining $\Omega_{\lambda}$ parameters. However, in view of large number of absorption transitions taken into account and small rms values obtained show that the differences principally arise due to overlapping emissions and nonradiative decay.

\section{Conclusions}

The Judd-Ofelt parameters deduced from the absorption spectra of $\mathrm{Pr}^{3+}$ and $\mathrm{Dy}^{3+}$ doped in NASICON type phosphate glass, NTZP give rise to reliable branching ratios for the emission transitions as evidenced by the good matching between the experimental and calculated values. The laser efficiency factors, viz. branching ratios and stimulated emission cross sections of $\mathrm{Pr}^{3+}$ doped glass show the potentiality of the glass system as laser medium. The appearance of emission lines from ${ }^{3} P_{1}$ level of $\mathrm{Pr}^{3+}$ at room temperature are due to thermalization effects. The phonon assisted side band appearing in the $\mathrm{Eu}^{3+}$ excitation spectrum is due to $\mathrm{P}-\mathrm{O}$ vibrational modes. Large electron-phonon coupling strength in this system suggest strong interaction of rare earth ion with the local sites and high non-radiative decay. The emission transition of $\mathrm{Eu}^{3+},{ }^{5} D_{0} \rightarrow{ }^{7} F_{0}$ is indicative of 8-9 coordination of rare earth with the oxygen ligands. The ratios of hypersensitive to non-hypersensitive emission lines of $\mathrm{Eu}^{3+}$ (red/orange) as well as $\mathrm{Dy}^{3+}$ (yellow/blue), show that the $\mathrm{Eu}^{3+}$ ions are situated in more distorted crystal field compared to $\mathrm{Dy}^{3+}$ ions.

\section{Acknowledgements}

The financial help from the Department of Science and Technology, New Delhi is gratefully acknowledged. The 
help rendered by Mrs D Usha Rani in preparation of glasses is appreciated.

\section{Appendix}

Judd-Ofelt parameters were calculated corresponding to the electronic transitions to the ${ }^{3} P_{0}$ level using branching ratios observed from the emission spectrum of $\mathrm{Pr}^{3+}$ and the oscillator strength of the ${ }^{3} H_{4} \rightarrow{ }^{3} P_{0}$ absorption transition according to Bunvel et al (1992).

Table. Oscillator strengths and Judd-Ofelt parameters of $\mathrm{Pr}^{3+}$ in NTZP glass. The observed values are obtained from absorption and emission spectra.

\begin{tabular}{lccc}
\hline \multirow{2}{*}{$\begin{array}{l}\mathrm{Pr}^{3+} \\
\text { transition }\end{array}$} & $\begin{array}{l}\text { Energy } \\
\left(\mathrm{cm}^{-1}\right)\end{array}$ & obs & cal \\
\cline { 3 - 4 } & 5197 & 2.87 & 2.29 \\
\hline${ }^{3} H_{4} \rightarrow{ }^{3} F_{2}$ & 6554 & 5.05 & 6.20 \\
${ }^{3} H_{4} \rightarrow{ }^{3} F_{3}$ & 7092 & 1.56 & 3.69 \\
${ }^{3} H_{4} \rightarrow{ }^{3} F_{4}$ & 9038 & 0.50 & 0.28 \\
${ }^{3} H_{4} \rightarrow{ }^{1} G_{4}$ & 16926 & 1.41 & 1.08 \\
${ }^{3} H_{4} \rightarrow{ }^{1} D_{2}$ & 20734 & 2.61 & 2.53 \\
${ }^{3} H_{4} \rightarrow{ }^{3} P_{0}$ & 21267 & 3.58 & 3.86 \\
${ }^{3} H_{4} \rightarrow{ }^{3} P_{1}$ & 22512 & 7.47 & 3.62 \\
${ }^{3} H_{4} \rightarrow{ }^{3} P_{2}{ }^{*}$ & 18182 & 0.37 & 0 \\
${ }^{3} H_{5} \rightarrow{ }^{3} P_{0}^{*}$ & 16239 & 1.41 & 1.17 \\
${ }^{3} H_{6} \rightarrow{ }^{3} P_{0}^{*}$ & 15537 & 0.19 & 0.34 \\
${ }^{3} F_{2} \rightarrow{ }^{3} P_{0}^{*}$ & & & \\
$\Omega_{2}=0.40 \times 10^{-20} \mathrm{~cm}^{2}$ & & & \\
$\Omega_{4}=3.87 \times 10^{-20} \mathrm{~cm}^{2}$ & & & \\
$\Omega_{6}=5.46 \times 10^{-20} \mathrm{~cm}^{2}$ & & rms = 1.42 \\
\hline
\end{tabular}

*hypersensitive transition.

"calculated from emission spectrum of $\mathrm{Pr}^{3+}$.

\section{References}

Binnemans $\mathrm{K}$ and Gorller-Walrand C $1998 \mathrm{~J}$. Phys. Condens. Matter 10 L167

Boulon G, Bouderbala $\mathrm{M}$ and Seriot J $1985 \mathrm{~J}$. Less-Common Metals 11241
Bunvel M A, Cases R, Chamano M A and Alcala R 1992 Phys. Chem. Glasses 3316

Caird J A, Ramponi A J and Staver P R 1991 J. Opt. Soc. Am. B8 1391

Camall W T, Hessler Jan P and Wagner F Jr 1978 J. Phys. Chem. 822152

Cho Doo-Hee, Hirao Kazuyki, Fujita Koji and Soga Naohiro 1996 J. Am. Ceram. Soc. 79327

Durville Frederic $\mathbf{M}$, Behrens Edward $\mathbf{G}$ and Powell Richand $\mathrm{C}$ 1987 Phys. Rev. B35 4109

Hagman L and Kierkegaard P 1968 Acta Chem. Scand. 221822

Menezes L de S, Cid b de Araujo, Maciel G S, Messadeq Y and Aegerter M A 1997 Appl. Phys. Lett. 70683

Nachimuthu P 1996 Optical properties of lanthanide ions doped in borate, phosphate and oxyfluoride glasses, $\mathrm{Ph} D$ thesis, University of Hyderabad, Hyderabad

Nachimuthu $P$ and Jagannathan R 1995a Phys. Chem. Glasses 3677

Nachimuthu P and Jagannathan R 1995b Proc. Indian Acad. Sci. (Chem. Sci.) 10759

Nachimuthu P, Jagannathan R, Nirmal Kumar V and Narayana Rao D 1997 J. Non-Cryst. Solids 217215

Reisfeld R 1975 Struct. and Bond 22123 and references therein Richardson F S, Saxe J D, Davis S A and Faulkner T R 1981 Molec. Phys. 421401

Sobha K C and Rao K J 1995a Solid State lonics 81145

Sobha K C and Rao K J 1995b Proc. Indian Acad. Sci. (Chem. Sci.) 107573

Sobha K C and Rao K J 1996a J. Non-Cryst. Solids 20152

Sobha K C and Rao K J 1996b J. Phys. Chem. Solids 571263

Srikanth Sarma P R, Jagannathan R and Vithal M $1992 \mathrm{~J}$. Chem. Soc. Dalton Trans. 723

Strek W, Szafranski C, Deren P, Jablonski K and JezowskaTrzebiaatowska B 1985 Rare earth spectroscopy (eds) B Jezowska-Trzebiaatowska, J Legendziewicz and W Strek (Philadelphia: World Scientific) p. 340

Su Qiang, Pei Zhiwu, Chi Lisheng, Zang Hongie, Zhang Zhongyi and Zou Feng $1993 \mathrm{~J}$. Alloys \& Comp. 19225

Tanabe S, Hirao K and Soga N 1992 J. Non-Cryst. Solids 142 148

Toratoni H, Jzumitani T and Kuroda H $1982 \mathrm{~J}$. Non-Cryst. Solids $\mathbf{5 2} 303$

Videau J J, Portier J and Pirion V $1982 \mathrm{~J}$. Non-Cryst. Solids 48385

Vijaya Prakash G, Nachimuthu P, Vithal $M$ and Jagannathan $R$ 1998 unpublished

Wei K, Machewirth D P, Wenzel J, Snitzer E and Sigel Jr G H 1994 Opt. Lett. 19904

Yzexing Zhao 1995 Opt. Lett. 20566

Zhao Yuzing and Flemming Simon 1997 IEEE J. QE 33905 